\title{
TRIB3 promotes oral squamous cell carcinoma cell proliferation by activating the AKT signaling pathway
}

\author{
PENG SHEN $^{1}$, TIAN-YANG ZHANG ${ }^{2}$ and SHU-YAN WANG ${ }^{3}$ \\ ${ }^{1}$ Department of Stomatology, Clinical Center of Spaceport, the Northern Medical District, Chinese People's Liberation Army \\ General Hospital, Beijing 100094; ${ }^{2}$ The 7 th Medical Center, Chinese People's Liberation Army General Hospital, Beijing \\ 100700; ${ }^{3}$ State Key Laboratory of Military Stomatology and National Clinical Research Center for Oral Diseases \\ and Shaanxi Clinical Research Center for Oral Diseases, School of Stomatology, \\ The Fourth Military Medical University, Xi'an, Shaanxi 710032, P.R. China
}

Received July 30, 2019; Accepted May 15, 2020

DOI: $10.3892 /$ etm.2021.9744

\begin{abstract}
Tribbles pseudokinase 3 (TRIB3), a member of the tribbles-related family, has biological roles such as by acting as an oncogene or tumor suppressor gene, in various types of cancer, including colorectal cancer, breast cancer, lung cancer and renal cell carcinoma. However, the role of TRIB3 in oral squamous cell carcinoma (OSCC) is remains unclear. The current was aimed to determine the biological function of TRIB3 in OSCC progression. TRIB3 expression was examined in OSCC surgical specimens using reverse transcription-quantitative PCR and the role of TRIB3 in the proliferation capacities of OSCC cell lines was examined using crystal violet and MTT assays in vitro and tumorigenicity assays in vivo. The underlying mechanism by which TRIB3 exerts its function was investigated using western blotting. The results demonstrated that the mRNA and protein expression levels of TRIB3 were higher in human OSCC tissues compared with normal tissues. The role of TRIB3 in cell proliferation was also determined. TRIB3 overexpression significantly promoted OSCC cell proliferation, whereas TRIB3 knockdown inhibited OSCC cell proliferation compared with control cells. TRIB3 knockdown also suppressed tumor growth and decreased tumor volume in vivo compared with control cells. Moreover, the results suggested that TRIB3 overexpression increased the phosphorylation of protein kinase B (AKT) and mammalian target of rapamycin (mTOR), whereas TRIB3 knockdown decreased the phosphorylation of AKT and mTOR compared with control cells. To summarize, the present study indicated that TRIB3 promoted OSCC cell proliferation by activating
\end{abstract}

Correspondence to: Dr Peng Shen, Department of Stomatology, Clinical Center of Spaceport, the Northern Medical District, Chinese People's Liberation Army General Hospital, 100 Youyi Road, Haidian, Beijing 10094, P.R. China

E-mail: sppeking@163.com

Key words: tribbles pseudokinase 3, proliferation, oral squamous cell carcinoma, AKT the AKT signaling pathway; therefore, TRIB3 may serve as a potential target for the diagnosis and treatment of OSCC.

\section{Introduction}

Oral squamous cell carcinoma (OSCC) develops from the epithelium lining of the oral cavity and accounts for $>90 \%$ of all cases of oral cancer worldwide (1). OSCC is the sixth most common solid tumor malignancy worldwide and is the most common malignant epithelial neoplasm occurring in the head and neck region in terms of incidence and mortality $(2,3)$. OSCC has a tendency to cause regional lymph node metastasis and recurrence (4). Despite advances in diagnostic and therapeutic strategies in the past few decades, the 5-year survival rate for OSCC remains relatively low at $\sim 50 \%$, which poses a great challenge for the prognosis of $\operatorname{OSCC}(5,6)$. Therefore, identifying the complicated molecular signatures of OSCC may aid in the diagnosis and treatment of the disease.

As a member of the tribbles-related family, Tribbles pseudokinase 3 (TRIB3) contains substrate-binding domains but lacks the conserved catalytic amino acid motifs essential for kinase activity $(7,8)$. A number of studies have demonstrated that TRIB3 is involved in diverse cellular processes, including cell proliferation and differentiation, the cellular stress response, epithelial-to-mesenchymal transition and glucose and lipid metabolism (9-11). Emerging evidence suggests that TRIB3 is a crucial oncoprotein, with the function of TRIB3 being associated with multiple different types of cancer, including breast, colorectal and lung cancer, as well as renal cell carcinoma (RCC) (12-15); however, the role of TRIB3 in OSCC is not completely understood. Additionally, the protein kinase $\mathrm{B}$ (AKT)/mammalian target of rapamycin (mTOR) signaling pathway has been determined to serve an important role in multiple cancers, including ovarian cancer, hepatocelluar carcinoma, lung cancer and OSCC (16).

The present study focused on the importance of TRIB3 during the progression of OSCC. TRIB3 was highly expressed in human OSCC tissues at both the mRNA and protein levels. Furthermore, the results demonstrated the significance of TRIB3 in the proliferation of OSCC cell lines in vitro and 
in vivo. Additionally, the molecular mechanism underlying TRIB3-mediated OSCC cell proliferation was investigated.

\section{Materials and methods}

OSCC tissue samples. After obtaining written informed consent from patients, a total of 35 primary OSCC tissues and paired adjacent normal tissues were obtained. All paired normal tissues were adjacent to tumor tissues at a distance $<0.5 \mathrm{~cm}$. All OSCC tissues were confirmed by two independent pathologists. RNA was extracted from 30 paired samples for analysis via reverse transcription-quantitative PCR (RT-qPCR) and five paired samples were assessed via western blotting. All patients (age, 45-62 years; mean age, 51 years; 21 male; 14 female) underwent surgery without chemotherapy or pre-operative radiation at the Chinese People's Liberation Army General Hospital (Beijing) from January 2017 to January 2019. The specimens were stored at $-80^{\circ} \mathrm{C}$ until further analysis. The present study was approved by the Institutional Review Board of the Chinese People's Liberation Army General Hospital.

Online microarray data. The relative transcript expression levels of TRIB3 and survival analyses were obtained from the Gene Expression Profiling Interactive Analysis (GEPIA; gepia. cancer-pku.cn), an online visual tool based on The Cancer Genome Atlas (TCGA) database (17). The online analysis of TRIB3 in GEPIA was based on the gene expression RNAseq dataset of head and neck squamous cell carcinoma (HNSC) in TCGA (tcga.xenahubs.net/download/TCGA.HNSC. sampleMap/HiSeqV2.gz), which included 44 normal tissues and 520 tumor tissues. The median value (8.1) of TRIB3 expression in tumour tissues was set as the cut-off value to divide the 520 patients with HNSC into two groups: TRIB3-low/medium group $(n=260)$ and TRIB3-high group $(n=260)$.

$R N A$ isolation and RT-qPCR. Total RNA was extracted from the tissues of 30 patients using TRIzol ${ }^{\circledR}$ reagent (Invitrogen; Thermo Fisher Scientific, Inc.) according to the manufacturer's instructions. High-quality RNA ( $2 \mu \mathrm{g})$ was reverse transcribed into cDNA using PrimeScript ${ }^{\mathrm{TM}}$ RT Reagent kit (Promega Corporation), according to the manufacturer's protocol. Subsequently, mRNA levels were quantified via qPCR using the PIKOREAL96 detection system (Thermo Fisher Scientific, Inc.) and the 2X SYBR Green kit (Roche Applied Science), according to the manufacturer's protocol. The following thermocycling conditions were used for qPCR: Initial denaturation for at $95^{\circ} \mathrm{C}$ for $1 \mathrm{~min}, 40$ cycles of denaturation for at $95^{\circ} \mathrm{C}$ for $20 \mathrm{sec}$, annealing at $60^{\circ} \mathrm{C}$ for $30 \mathrm{sec}$ and extension at $72^{\circ} \mathrm{C}$ for $20 \mathrm{sec}$, followed by final extension at $72^{\circ} \mathrm{C}$ for $10 \mathrm{~min}$. The following primers were used for qPCR: TRIB3 forward, 5'-AAGCGGTTGGAGTTGGATGAC-3' and reverse, 5'-CAC GATCTGGAGCAGTAGGTG-3'; and GAPDH forward, 5'-ATGACCCCTTCATTGACCTCA-3' and reverse, 5'-GAG ATGATCACCCTTTTGGCT-3'. TRIB3 mRNA expression levels were quantified using the $2^{-\Delta \Delta \mathrm{Cq}}$ method (18) and normalized to the internal reference gene GAPDH.

Cell culture. OSCC (SCC9, HSC3, Cal27 and SCC15), normal oral (HOK) and 293T cell lines were purchased from The
Cell Bank of Type Culture Collection of Chinese Academy of Sciences. All cell lines were maintained in DMEM medium (Invitrogen; Thermo Fisher Scientific, Inc.) supplemented with 10\% FBS (Invitrogen; Thermo Fisher Scientific, Inc.) and 1\% penicillin/streptomycin (Sangon Biotech Co., Ltd.) at $37^{\circ} \mathrm{C}$ in humidified atmosphere containing $5 \% \mathrm{CO}_{2}$.

Cell transfection. A TRIB3 coding sequence was constructed and inserted into the p23-3xflag-GFP vector (Invitrogen; Thermo Fisher Scientific, Inc.) to generate the TRIB3 overexpression vector. Lentiviral short hairpin (sh)RNAs targeting TRIB3 were designed and constructed into the pLKO.1-TRC vector (Sangon Biotech Co., Ltd.) A non-targeted scrambled (SCR) oligonucleotide (Sangon Biotech Co., Ltd.) was used as the negative control.

To produce lentiviral particles for TRIB3 overexpression and knockdown, the core plasmid $(1.0 \mu \mathrm{g})$ was co-transfected with the packaging plasmids pMD2.G and psPAX2 $(2.5 \mu \mathrm{g}$, Sangon Biotech Co., Ltd) into $293 \mathrm{~T}$ cells $\left(5 \times 10^{5}\right.$ units/well) using a calcium phosphate co-precipitation method (19). At $12 \mathrm{~h}$ post-transfection, the medium was replaced with DMEM. At $24 \mathrm{~h}$ post-transfection, the supernatants containing the virus were collected and filtered through a $0.45 \mu \mathrm{m}$ membrane. Subsequently, the virus was concentrated by centrifugation at $50,000 \mathrm{xg}$ for $140 \mathrm{~min}$ at $4^{\circ} \mathrm{C}$. The pellet was resuspended in $1.5 \mathrm{ml}$ EP tube (Sangon Biotech Co., Ltd.), aliquoted and stored at $-80^{\circ} \mathrm{C}$ until further use. For the transduction process, HSC 3 and Cal27 cells were grown to $60 \%$ confluence in 6-well plates and transfected with $30 \mu \mathrm{l}$ virus supernatant $\left(\sim 5 \times 10^{6}\right.$ units per well $)$ with $5 \mu \mathrm{g} / \mathrm{ml}$ polybrene (Hanheng Biological Technology Co., Ltd.). After $24 \mathrm{~h}$ at $37^{\circ} \mathrm{C}$, the medium was replaced and the cells were transferred to $10-\mathrm{cm}$ dishes. After 2 days at $37^{\circ} \mathrm{C}$, TRIB3-overexpression HSC 3 and Cal27 cells were screened by green fluorescence via flow cytometry using an IX-71 flow cytometer (Olympus Corporation). TRIB3-knockdown SCC9 and SCC15 cells were cultured and screened in medium containing $4 \mu \mathrm{g} / \mathrm{ml}$ puromycin (Hanheng Biological Technology Co., Ltd.) for 4 days. Subsequently, individual puromycin-resistant colonies were isolated. Transfection efficiency was verified by western blotting. Subsequent experiments were performed $24 \mathrm{~h}$ after transfection. The sequences of the SCR were: Forward, 5'-TTC TCCGAACGTGTCACGT-3' and reverse, 5'-ACGTGACAC GTTCGGAGAA-3'. The sequences of the shTRIB3 were: Forward, 5'-CCGGGATCTCAAGCTGTGTCGCTTTCT CGAGAAAGCGACACAGCTTGAGATCTTTTTG-3' and reverse, 5'-AATTCAAAAAGATCTCAAGCTGTGTCGCTT TCTCGAGAAAGCGACACAGCTTGAGATC-3'.

Western blotting. Total protein was extracted from paired tumor tissues and cell lines by lysing cells in RIPA buffer (Thermo Fisher Scientific, Inc.) for $\geq 30 \mathrm{~min}$ on ice. Cell lysates were centrifuged at $10,000 \mathrm{x}$ g for $15 \mathrm{~min}$ at $4^{\circ} \mathrm{C}$. Total protein was quantified using Bradford reagent (Sigma-Aldrich; Merck KGaA) according to the manufacturer's protocol. Proteins $(10 \mu \mathrm{g})$ were separated via $10 \%$ SDS-PAGE and transferred onto PVDF membranes. The membranes were blocked with 5\% fat-free milk for $1 \mathrm{~h}$ at room temperature. Subsequently, the membranes were incubated at $4^{\circ} \mathrm{C}$ overnight with primary antibodies targeted 
A

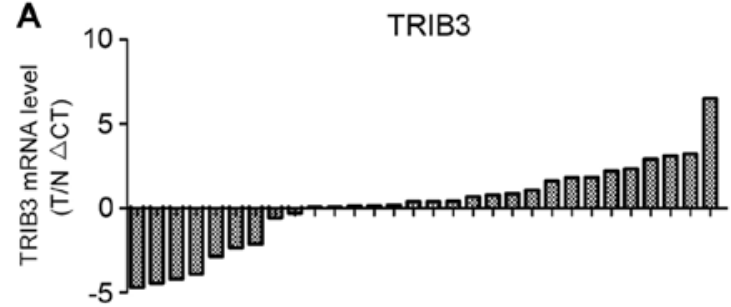

C

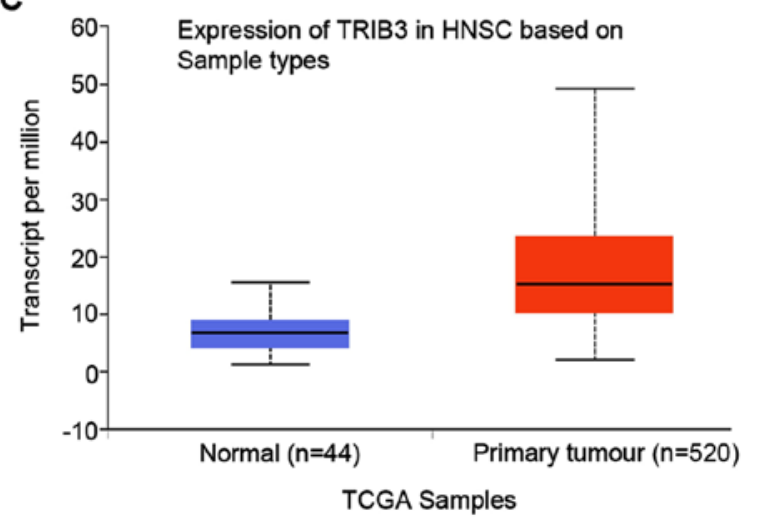

B

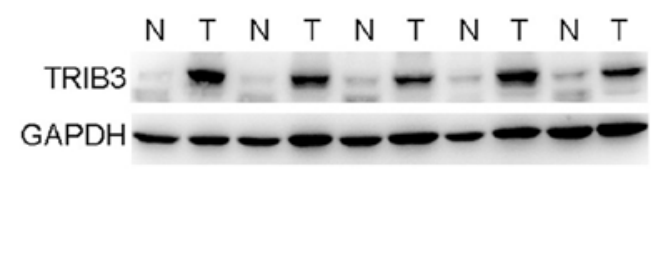

D

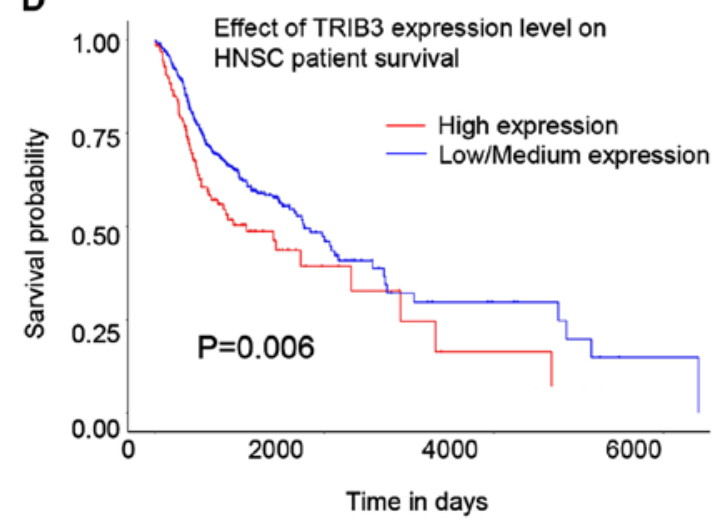

Figure 1. TRIB3 expression is higher in OSCC tissues and is associated with patient survival. (A) TRIB3 mRNA expression levels in 30 paired tumour tissues and corresponding normal tissues. (B) TRIB3 protein expression levels in 5 paired tumour tissues and corresponding normal tissues. (C) Based on the GEPIA database, TRIB3 expression was higher in tumor tissues compared with normal tissues. (D) Based on the GEPIA database, high TRIB3 expression was associated with worse overall survival rates in patients with OSCC (from GEPIA database). TRIB3, tribbles pseudokinase 3; OSCC, oral squamous cell carcinoma; GEPIA, Gene Expression Profiling Interactive Analysis; N, normal; T, tumour; HNSC, head-neck squamous cell carcinoma; TCGA, The Cancer Genome Atlas.

against: TRIB3 (cat. no. ab137526; 1:1,000; Abcam), AKT (cat.no. 4691; 1:1,000; Cell Signaling Technology, Inc.), phosphorylated (p)-AKT (cat. no. 4060; 1:1,000; Cell Signaling Technology, Inc.), mTOR (cat. no. 2983; 1:1,000; Cell Signaling Technology, Inc.), p-mTOR (cat. no. 5536; 1:1,000; Cell Signaling Technology, Inc.), Flag (cat. no. 8164; 1:1,000; Cell Signaling Technology, Inc.) and GAPDH (cat. no. 5174; 1:1,000; Cell Signaling Technology, Inc.). Following primary incubation, the membranes were incubated with an anti-rabbit horseradish peroxidase-conjugated secondary antibody (cat. no. D110103; 1:5,000; Sangon Biotech Co., Ltd.) for $2 \mathrm{~h}$ at room temperature. Immunoreactive protein bands were visualized using an enhanced chemiluminescence kit (Pierce; Thermo Fisher Scientific, Inc.) and a Gel Dox XR system (Bio-Rad Laboratories, Inc.). GAPDH was used as the loading control. ImageJ software (version 1.8.0, National Institutes of Health) was used for quantification.

Immunohistochemistry. Tumor xenografts were fixed in $4 \%$ formalin (Sangon Biotech Co., Ltd.) at room temperature for $12 \mathrm{~h}$, embedded in paraffin and cut into 5- $\mu \mathrm{m}$-thick consecutive sections. After deparaffinization and antigen recovery in a sodium citrate solution ( $\mathrm{pH}$ 6.0) for $20 \mathrm{~min}$ at $98^{\circ} \mathrm{C}$, the sections were washed three times with $0.01 \mathrm{~mol} / 1 \mathrm{PBS}$ for $5 \mathrm{~min}$ each time and blocked for $1 \mathrm{~h}$ in $0.01 \mathrm{~mol} / 1 \mathrm{PBS}$ containing 0.3\% Triton X-100 and 5\% BSA (Gibco; Thermo Fisher Scientific, Inc.). Subsequently, the sections were incubated with anti-TRIB3 (cat. no. ab137526; 1:200; Abcam) and anti-p-AKT (cat. no. 4060; 1:200; Cell Signaling Technology, Inc.) primary antibodies at $4^{\circ} \mathrm{C}$ overnight. After washing with
$0.01 \mathrm{~mol} / 1 \mathrm{PBS}$, the sections were incubated with $0.01 \mathrm{~mol} / 1$ PBS containing a horseradish peroxidase-conjugated anti-rabbit IgG secondary antibody (cat. no. 7074; 1:5,000; Cell Signaling Technology, Inc.) at room temperature for $2 \mathrm{~h}$. Subsequently, the sections were developed using $0.003 \%$ $\mathrm{H}_{2} \mathrm{O}_{2}$ and $0.03 \%$ 3,3'-diaminobenzidine in $0.05 \mathrm{~mol} / 1$ Tris- $\mathrm{HCl}$ (pH 7.6). Immunohistochemistry was performed in triplicate. Stained sections were observed in five randomly selected and independent high-power microscopic fields of view using an inverted light microscope (magnification, x400).

Crystal violet assay. Cells were seeded $\left(1 \times 10^{3}\right.$ cells/well) into 6-well plates and cultured in DMEM medium supplemented with $10 \%$ FBS. The medium was replaced every three days. After 2 weeks, the medium was removed and the cells were fixed with $20 \%$ methanol at room temperature for $10 \mathrm{~min}$. Subsequently, the cells were stained with $0.5 \%$ crystal violet (Sigma-Aldrich; Merck KGaA) at room temperature or $10 \mathrm{~min}$. Cells were washed with PBS and photographed using a light microscope (magnification, $\mathrm{x} 4$ ). The absorbance values were measured at a wavelength of $600 \mathrm{~nm}$ using a microplate reader.

MTT assay. Cells were seeded $\left(1 \times 10^{3}\right.$ cells/well) into 96 -well plates. Subsequently, $20 \mu \mathrm{l}$ MTT solution $(5 \mathrm{mg} / \mathrm{ml})$ was added to each well and incubated at $37^{\circ} \mathrm{C}$ for $4 \mathrm{~h}$. The medium was then removed and $200 \mu \mathrm{l}$ DMSO was added to dissolve the formazan crystals. The microtiter plate was placed on a shaker to dissolve the crystals. The absorbance of each well was measured at a wavelength of $490 \mathrm{~nm}$ using an automated microplate reader. The MTT assay was performed in triplicate. 


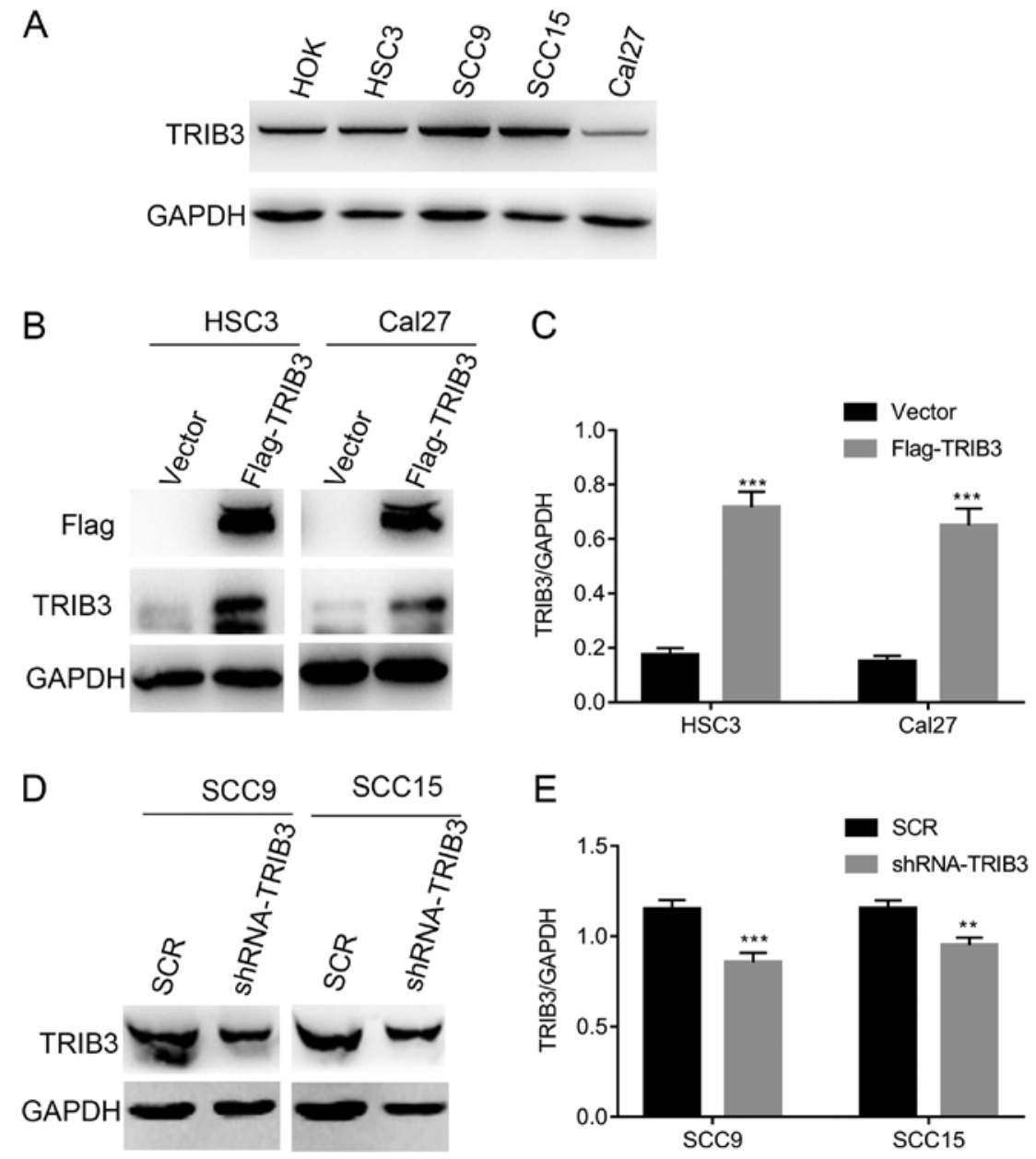

Figure 2. TRIB3 overexpression and knockdown in OSCC cell lines. (A) Endogenous TRIB3 expression levels in OSCC and normal oral cell lines were measured by western blotting. Flag and TRIB3 expression levels following TRIB3 overexpression in HSC3 and Cal27 cells were (B) determined by western blotting and (C) semi-quantified. TRIB3 expression levels following TRIB3 knockdown in SCC9 and SCC15 cells were (D) determined by western blotting and (E) semi-quantified. ${ }^{* *} \mathrm{P}<0.01$ and ${ }^{* * *} \mathrm{P}<0.001$ vs. vector/SCR. TRIB3, tribbles pseudokinase 3; OSCC, oral squamous cell carcinoma; SCR, scrambled; shRNA, short hairpin RNA.

Tumorigenesis in vivo. A total of 8 male BALB/c nude mice (age, 5 weeks; median weight, $19 \mathrm{~g}$; weight range, 18-21 g) were obtained from Beijing Huafukang Bioscience Co., Ltd. Mice were housed with free access to regular chow diet under specific pathogen-free conditions at $23 \pm 3^{\circ} \mathrm{C}$ with $35 \pm 5 \%$ humidity and 12-h light/dark cycles. raised under standard conditions. Mice were subcutaneously injected with $100 \mu \mathrm{l}$ cell suspension with PBS as the vehicle $\left(1 \times 10^{6}\right.$ cells $\left./ \mathrm{ml}\right)$ of SCR negative control or shRNA-TRIB3 SCC9 cells into the right flank. Mice in the SCC9 SCR group $(n=4)$ received an injection of SCR SCC9 cells and mice in the SCC9 shRNA-TRIB3 group $(n=4)$ received an injection of shRNA-TRIB3 SCC9 cells. Tumor volume was measured every 7 days according to the following equation: Volume $=0.5 \mathrm{x}$ length $\mathrm{x}$ width ${ }^{2}$. At the end of the experiment (5 weeks), the mice were sacrificed by cervical dislocation and tumors were excised. The tumors were photographed and weighed. The maximum tumor volume observed was $698 \mathrm{~mm}^{3}$. All animal experiments were approved by the Institutional Animal Care and Use Committee of the Chinese People's Liberation Army General Hospital.

Statistical analysis. Statistical analyses were performed using GraphPad Prism (version 5; GraphPad Software, Inc.). Data are presented as the mean \pm standard deviation. Comparisons between two groups were analyzed using the unpaired Student's t-test. All experiments were performed in triplicate. $\mathrm{P}<0.05$ was considered to indicate a statistically significant difference.

\section{Results}

TRIB3 is upregulated in OSCC tissues. Firstly, TRIB3 mRNA expression levels in 30 paired OSCC tissues and corresponding normal tissues were measured. TRIB3 mRNA expression was higher in 21/30 OSCC tissues compared with the corresponding normal tissues (Fig. 1A). In addition, TRIB3 protein expression levels were also higher in OSCC tissues compared with the corresponding normal tissues (Fig. 1B), which was consistent with the RT-qPCR results. As indicated by data obtained from the GEPIA database, TRIB3 expression was higher in the 520 tumor tissues compared with the 44 normal tissues (Fig. 1C). Furthermore, the overall survival rate of the TRIB3-low/medium group was significantly improved compared with the TRIB3-high group ( $\mathrm{P}=0.006$; Fig. 1D). Based on the expression analyses and the data obtained from the GEPIA database, the results indicated 
A

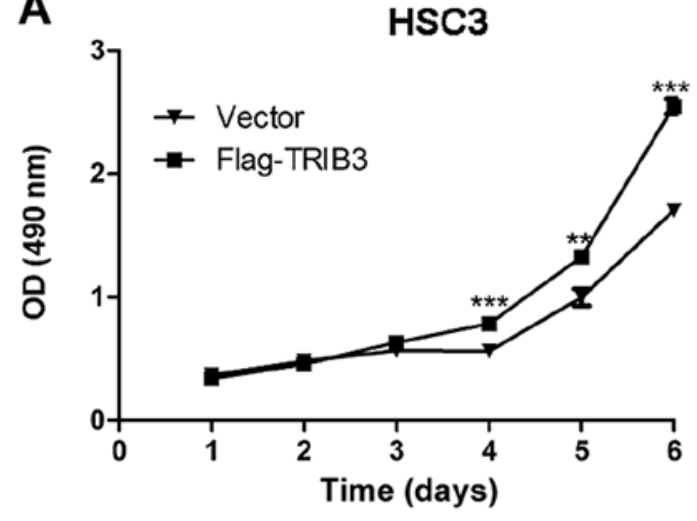

C

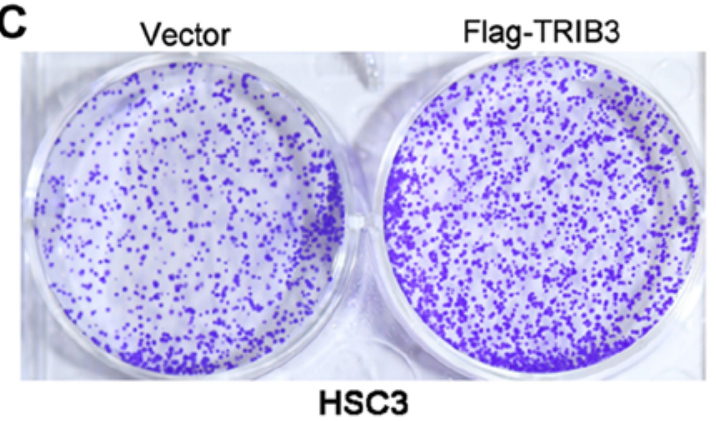

$\mathbf{E}$

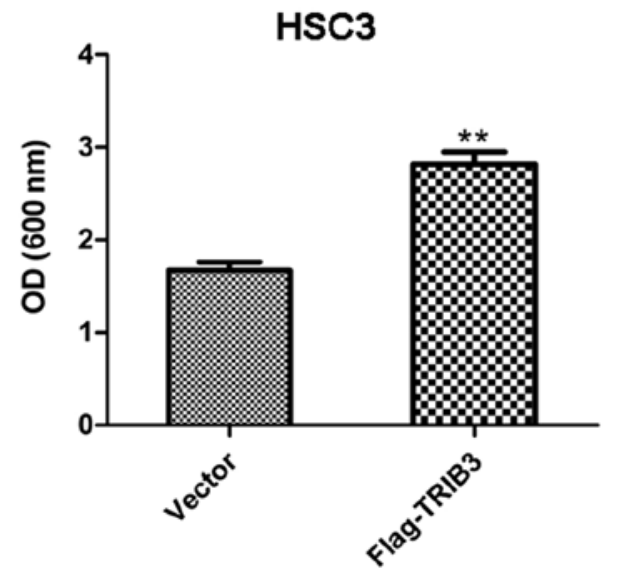

B

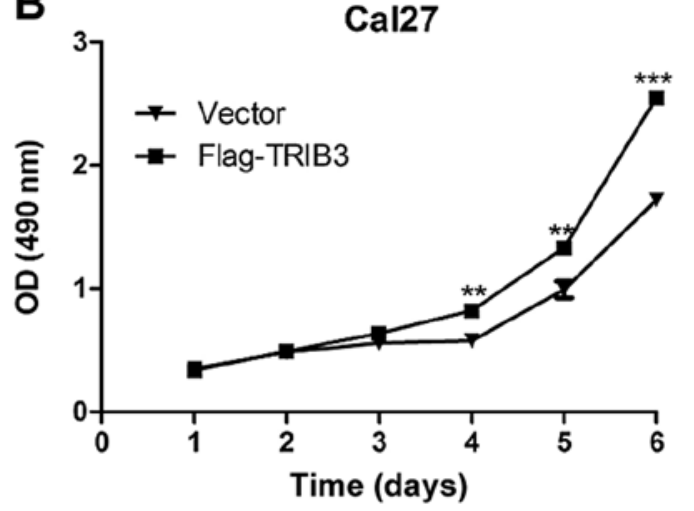

D

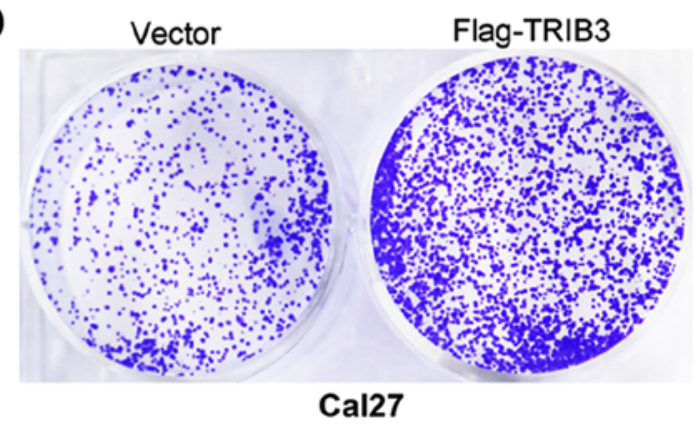

$\mathbf{F}$

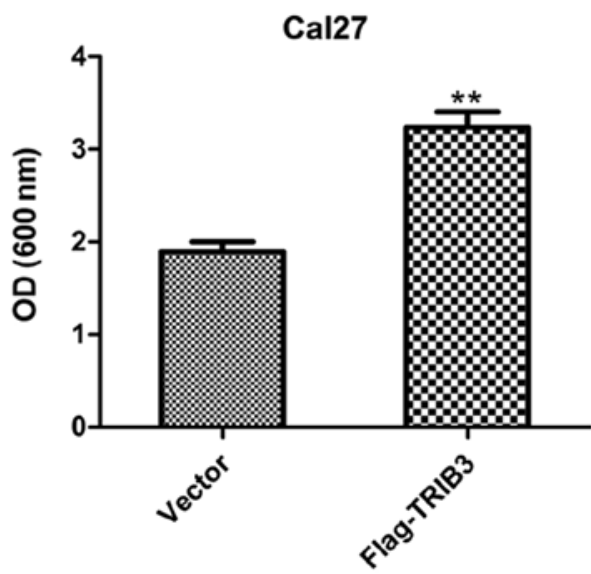

Figure 3. TRIB3 overexpression promotes OSCC cell proliferation. The effect of TRIB3 overexpression on (A) HSC3 and (B) Cal27 cell proliferation was assessed. The effect of TRIB3 overexpression on the colony formation ability of (C) HSC3 and (D) Cal27 cells was assessed (magnification, $\mathrm{x} 4)$. The absorbance values of the crystal violet assay in (E) HSC 3 and (F) Cal27 cells. ${ }^{* *} \mathrm{P}<0.01$ and ${ }^{* * *} \mathrm{P}<0.001$ vs. vector. TRIB3, tribbles pseudokinase 3 ; OSCC, oral squamous cell carcinoma; OD, optical density.

that TRIB3 was upregulated in OSCC tissues compared with normal tissues.

TRIB3 overexpression and knockdown in OSCC cell lines. Based on the results obtained from the clinical data, it was hypothesized that TRIB3 might influence OSCC cell proliferation. Firstly, endogenous TRIB3 expression levels in several human OSCC cell lines (SCC9, HSC3, Cal27 and SCC15) and a normal oral cell line (HOK) were investigated. TRIB3 expression was higher in SCC9 and SCC15 cells compared with HSC3, Cal27 and HOK cells (Fig. 2A). To identify the function of TRIB3 in OSCC cells, HSC3 and Cal27 cells were transduced with empty p23 vector or TRIB3 overexpression vector (Flag-TRIB3) plasmids. In addition, shRNA-TRIB3 was used to knockdown TRIB3 expression in SCC9 and SCC15 cell lines. Transfection efficiency was verified via western blotting (Fig. 2B-E).

TRIB3 overexpression promotes the proliferation of OSCC cell lines. The MTT assay indicated that HSC3 and Cal27 cell proliferation was significantly increased at 4,5 and 6 days post-transfection in the TRIB3 overexpression group compared with the untreated group ( $\mathrm{P}<0.01$; Fig. $3 \mathrm{~A}$ and $\mathrm{B})$. Furthermore, the crystal violet assay suggested that TRIB3 overexpression markedly promoted the colony formation ability of HSC 3 and Cal27 cells compared with the untreated 
A

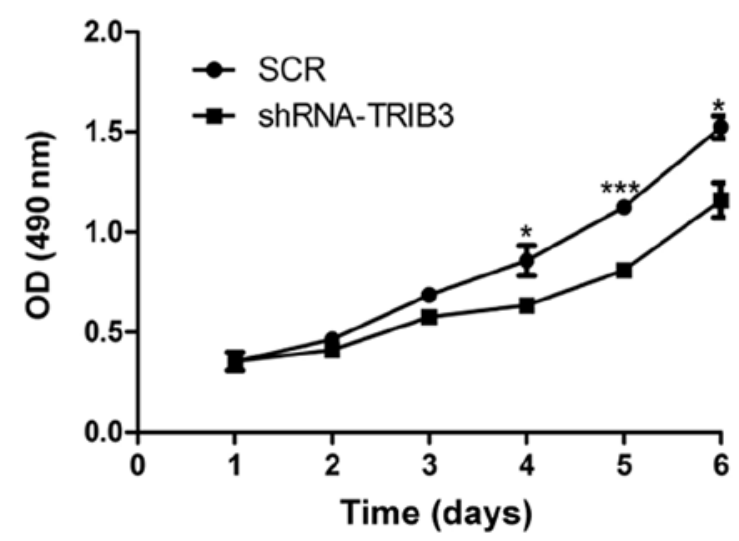

C

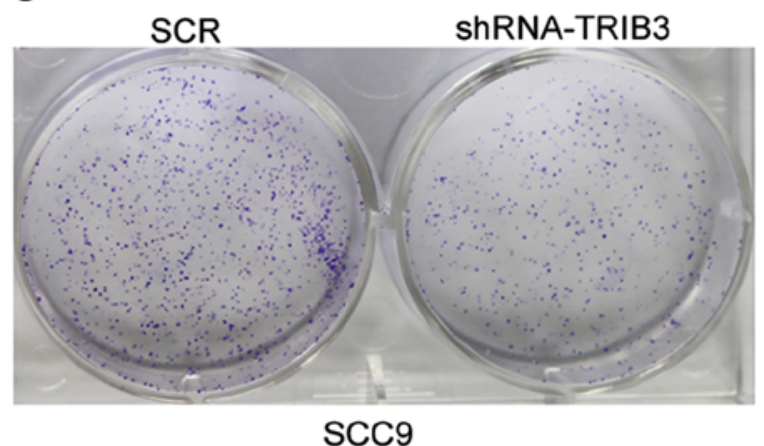

E

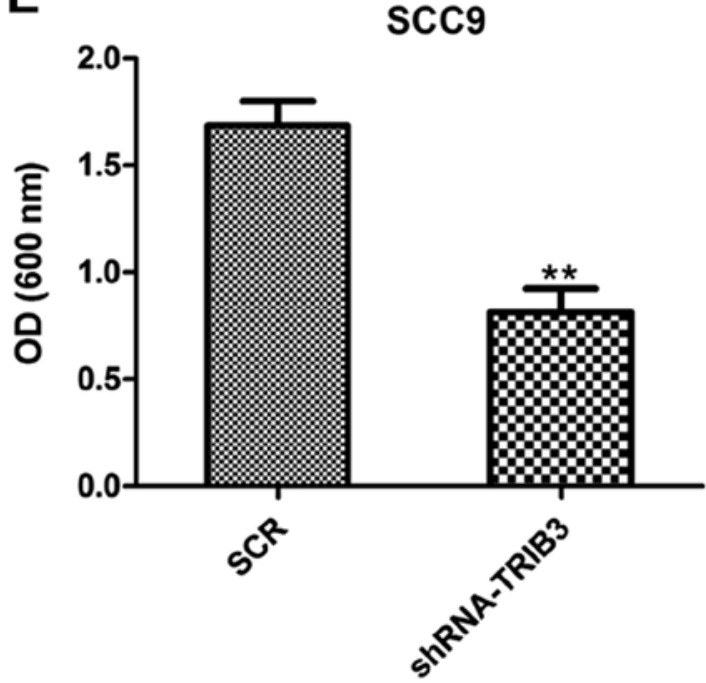

B $\operatorname{SCC15}$

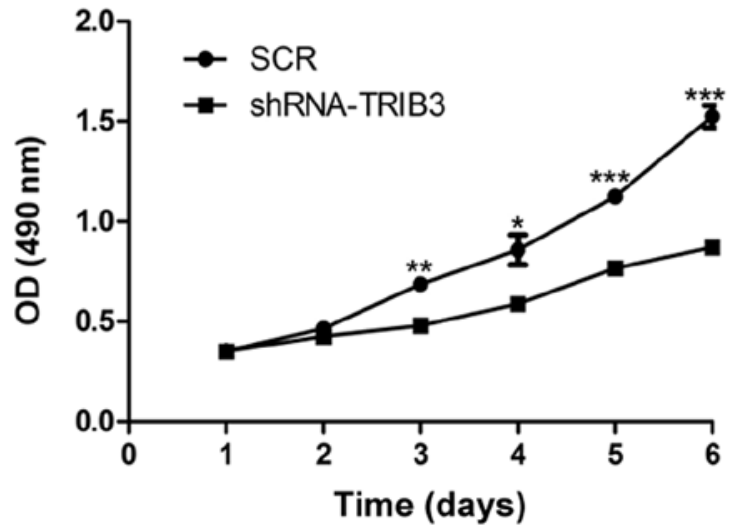

D

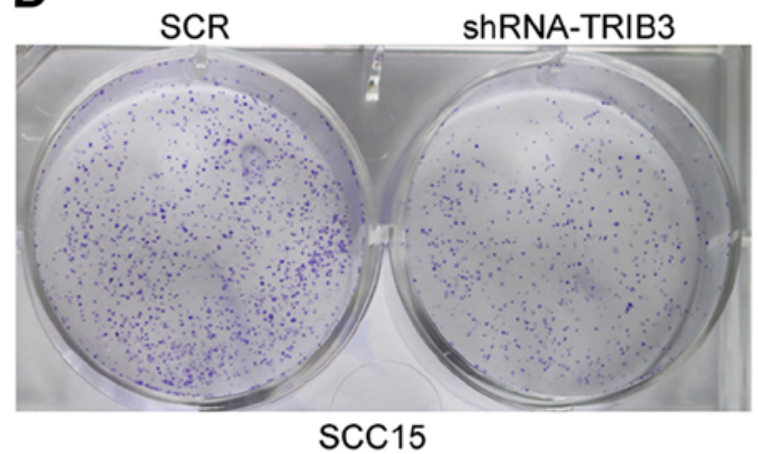

$\mathbf{F}$

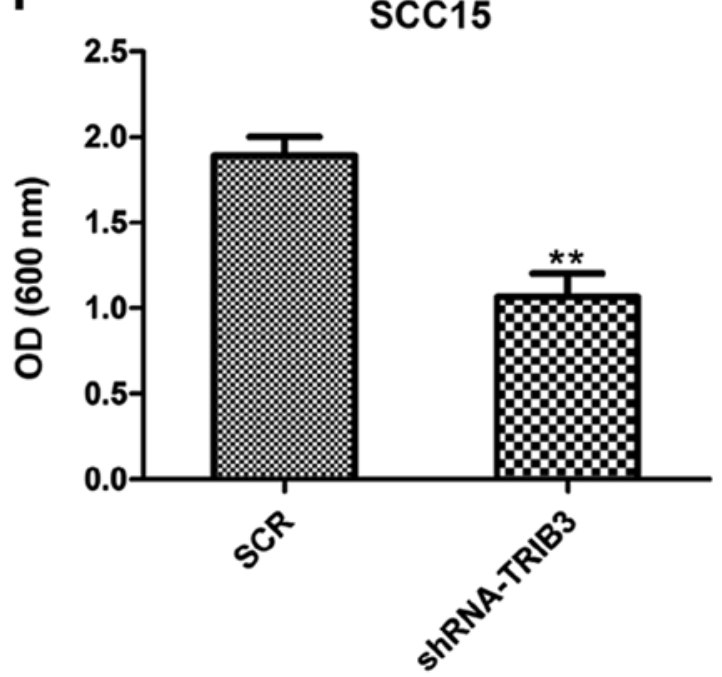

Figure 4. TRIB3 knockdown inhibits OSCC cell proliferation. The effect of TRIB3 knockdown on (A) SCC9 and (B) SCC15 cell proliferation was assessed. In addition, the effect of TRIB3 knockdown on the colony formation ability of (C) SCC9 and (D) SCC15 cells was assessed (magnification, x4). The absorbance values of the crystal violet assay in (E) SCC9 and (F) SCC15 cells. ${ }^{*} \mathrm{P}<0.05,{ }^{* *} \mathrm{P}<0.01$ and ${ }^{* * *} \mathrm{P}<0.001$ vs. SCR. TRIB3, tribbles pseudokinase 3; OSCC, oral squamous cell carcinoma; SCR, scrambled; OD, optical density.

group (Fig. 3C and D), which reflected the enhanced proliferative ability of the TRIB3-overexpression HSC 3 and Cal27 cells. Furthermore, the absorbance values of TRIB3-overexpression HSC3 and Cal27 cells were significantly higher compared with untreated cells $(\mathrm{P}<0.01$; Fig. 3E and F).

TRIB3 knockdown suppresses the proliferation of OSCC cell lines. TRIB3-knockdown SCC9 and SCC15 cell lines were established by transfection with shRNA-TRIB3 and a non-targeting control lentivirus (SCR). Similar to the overexpression experiments, the proliferation of the SCR- and shRNA-TRIB3-transfected cell lines was assessed. The MTT assay results indicated that SCC9 cell proliferation was significantly reduced in the TRIB3 knockdown group at 4,5 and 6 days post-transfection compared with the untreated group $(\mathrm{P}<0.05$; Fig. $4 \mathrm{~A})$. Similarly, $\mathrm{SCC} 15$ cell proliferation 

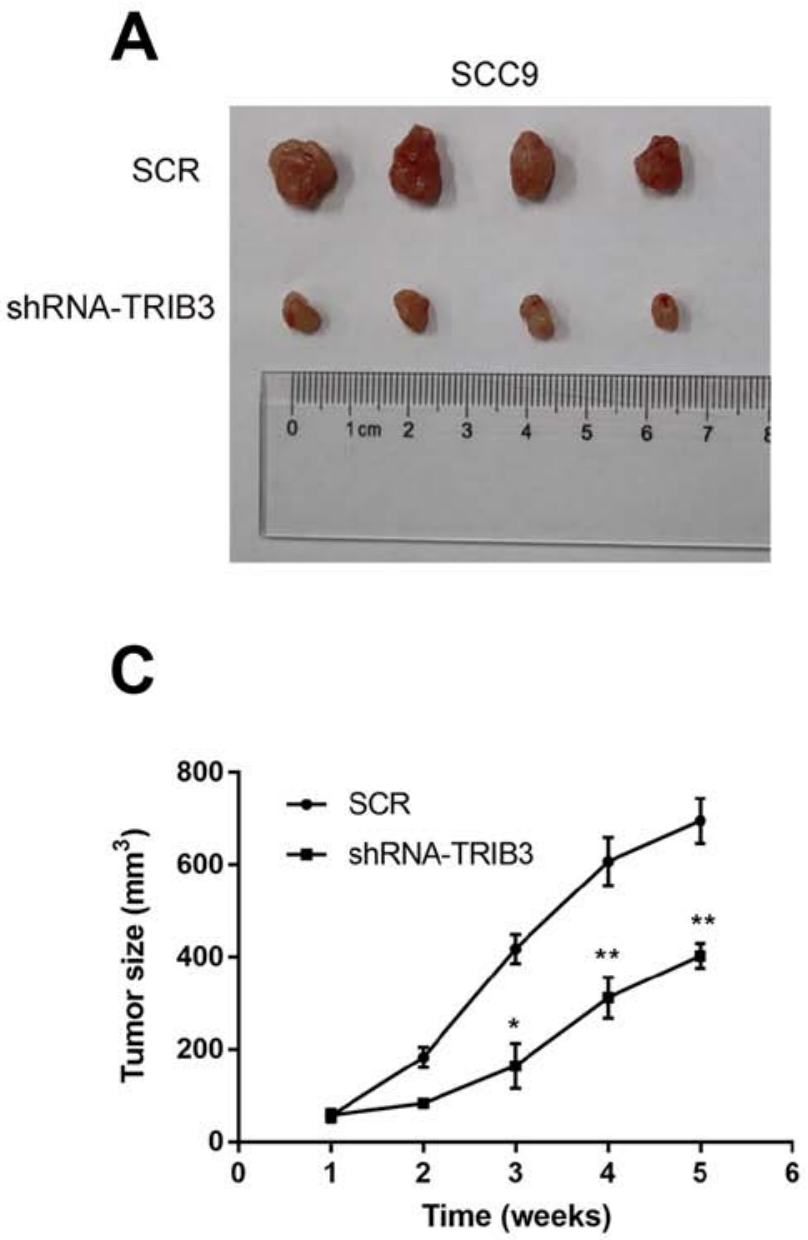

B

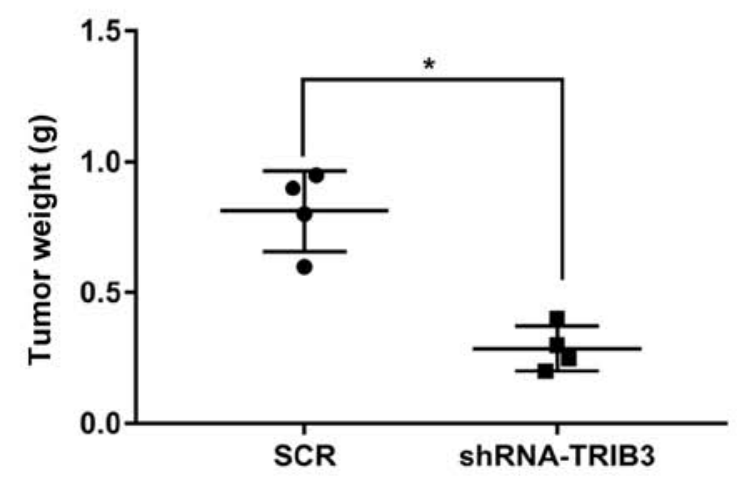

D

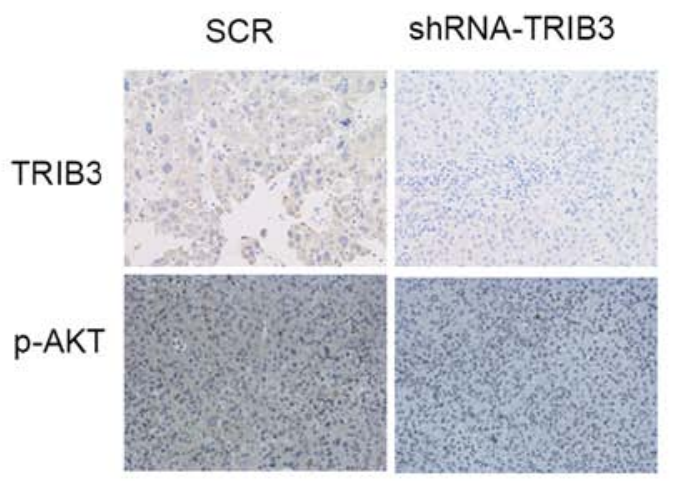

Figure 5. TRIB3 knockdown suppresses tumor development in vivo. (A) Representative images of tumors generated by control and TRIB3-knockdown SCC9 cells. (B) Tumour weight. (C) Tumour volume. (D) Immunohistochemical staining of TRIB3 and p-AKT in tumor xenografts (magnification, $\mathrm{x} 400$ ). * $\mathrm{P}<0.05$ and ${ }^{* *} \mathrm{P}<0.01$ vs. SCR. TRIB3, tribbles pseudokinase 3; p, phosphorylated; SCR, scrambled; shRNA, short hairpin RNA.

at 3, 4, 5 and 6 days post-transfection was significantly reduced in the TRIB3 knockdown group compared with the untreated group $(\mathrm{P}<0.05$; Fig. 4B). Furthermore, the crystal violet assay results indicated that the colony formation ability of SCC9 and SCC15 cells was significantly lower in TRIB3-knockdown cells compared with untreated cells $(\mathrm{P}<0.01$; Fig. 4C-F $)$.

TRIB3 knockdown suppresses OSCC cell tumorigenesis in vivo. To verify the in vitro results in vivo, control and TRIB3-knockdown SCC9 cells were subcutaneously injected into the right flank of nude mice and tumor growth was monitored (Fig. 5A). The mean tumor weight in the shRNA-TRIB3 group was significantly lower compared with the SCR group $(n=4 ; P=0.001$; Fig. 5B). Similarly, the mean tumor volume of the shRNA-TRIB3 group was significantly lower compared with the SCR group at 3,4 and 5 weeks post-injection $(n=4$; $\mathrm{P}<0.05$; Fig. 5C), which suggested that TRIB3 knockdown suppressed OSCC cell-mediated tumour growth in vivo. The results were consistent with the in vitro results, which indicated that TRIB3 knockdown inhibited OSCC cell proliferation. In addition, AKT phosphorylation was notably increased in the shRNA-TRIB3 group compared with the SCR group (Fig. 5D).

TRIB3 activates the AKT signaling pathway in OSCC cells. It has been reported that constitutive AKT activation serves an important role during the development and progression of OSCC (20). To elucidate the mechanism underlying TRIB3-mediated OSCC tumorigenesis, western blotting was performed to investigate the protein expression levels of p-AKT and total AKT in TRIB3 overexpression and knockdown cell lines. TRIB3 overexpression markedly increased AKT and mTOR phosphorylation levels in HSC3 and Cal27 cells compared with control cells, without notably altering the total AKT and mTOR expression levels (Fig. 6A and B). By contrast, TRIB3 knockdown in SCC9 and SCC15 cells notably reduced AKT and mTOR phosphorylation compared with SCR-transfected cells, without markedly altering the total AKT and mTOR expression levels (Fig. 6C and D). In summary, the results indicated that TRIB3 promoted AKT activation.

\section{Discussion}

In previous years, emerging evidence has indicated that TRIB3 is involved in tumorigenesis and cancer progression (12-15). Upregulated TRIB3 expression has been associated with a suboptimal prognosis in colon and breast cancer $(12,21)$. In addition, the expression level of TRIB3 was reported to display an inverse relationship with the prognosis of breast cancer (22). Conversely, TRIB3 upregulation was significantly 
A

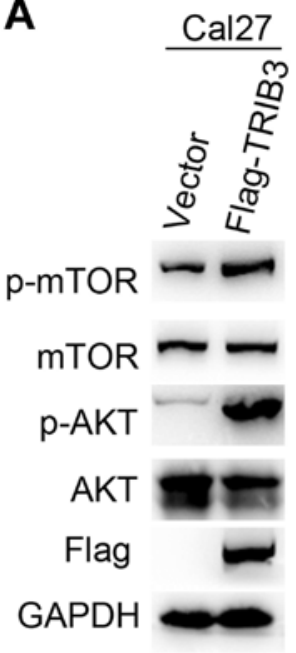

C

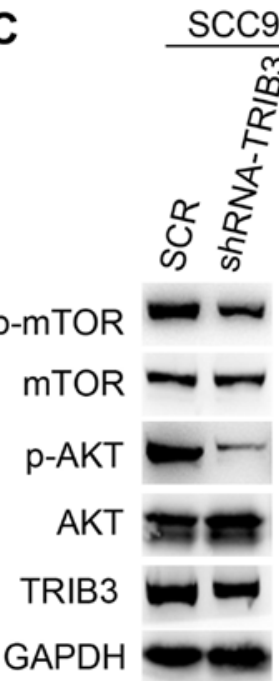

B
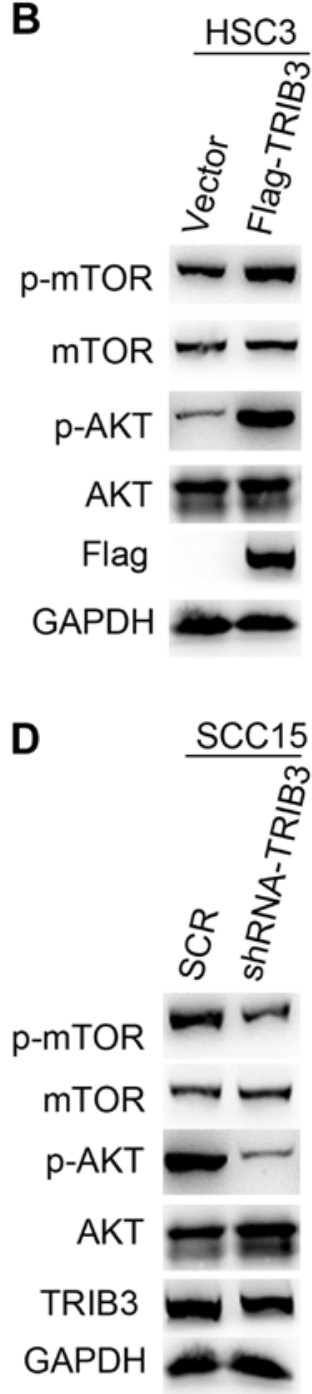

Figure 6. TRIB3 activates the AKT signaling pathway. TRIB3 overexpression activated the AKT signaling pathway in (A) HSC3 and (B) Cal27 cells. TRIB3 knockdown inhibited the AKT signaling pathway in (C) SCC9 and (D) SCC15 cells. TRIB3, tribbles pseudokinase 3; p, phosphorylated; SCR, scrambled; shRNA, short hairpin RNA.

correlated with tumor size and lymph node or distal metastasis in patients with non-small cell lung cancer (NSCLC), suggesting that TRIB3 upregulation was associated with the poor prognosis of NSCLC (13). Furthermore, Hong et al (15) demonstrated the potential oncogenic role of TRIB3 in RCC, reporting that TRIB3 promoted RCC cell proliferation, migration and invasion.

In the present study, TRIB3 mRNA and protein expression levels were markedly higher in human OSCC tissues compared with normal tissues. Cell proliferation was significantly enhanced in TRIB3-overexpression cells compared with control cells. In addition, TRIB3 knockdown inhibited OSCC cell proliferation compared with control cells. Moreover, TRIB3 knockdown suppressed tumor growth and decreased tumor volume in vivo compared with control cells. The mechanism underlying TRIB3-mediated OSCC tumorigenesis was also investigated. The results indicated that compared with control cells, AKT and mTOR phosphorylation was significantly increased following TRIB3 overexpression, whereas TRIB3 knockdown decreased AKT and mTOR phosphorylation, which is crucial for tumor progression (23). Based on the results, it was hypothesized that TRIB3 may promote OSCC cell proliferation by activating the AKT signaling pathway. The AKT signaling pathway serves an important role in multiple types of cancer, including ovarian cancer, hepatocelluar carcinoma and lung cancer $(24,25)$. The results of the present study were consistent with a previous study, which reported that TRIB3 inhibited tumorigenesis by targeting AKT (26). Restelli et al (26) demonstrated that TRIB3 could inhibit several cancer-related processes, such as cell proliferation and invasion, by binding to Ser473 of the AKT protein kinase. To the best of our knowledge, the present study was the first study to demonstrate the role of TRIB3 in OSCC cell proliferation. However, the present study had a number of limitations, including the lack of analysis of mTOR expression levels via immunohistochemistry, as well as the lack of prognostic analysis of TRIB3 in clinical OSCC samples by performing a tissue microarray.

In summary, the present study indicated that TRIB3 may serve a critical role in OSCC cell proliferation. The results may improve the current understanding of the mechanisms underlying the biological role of TRIB3 during tumor development and might provide a potential therapeutic target for OSCC.

\section{Acknowledgements}

Not applicable.

\section{Funding}

No funding was received.

\section{Availability of data and materials}

The datasets generated and/or analyzed during the current study are available in the tcga.xenahubs.net/download/TCGA. HNSC.sampleMap/HiSeqV2.gz.

\section{Authors' contributions}

PS and TYZ conducted the experiments. PS designed the study. SYW analyzed data. PS and SYW drafted the manuscript. All authors read and approved the final manuscript.

\section{Ethics approval and consent to participate}

The present study was approved by the Institutional Review Board of the Chinese People's Liberation Army General Hospital (Beijing, China). Written informed consent was obtained from all subjects.

\section{Patient consent for publication}

Not applicable.

\section{Competing interests}

The authors declare that they have no competing interests. 


\section{References}

1. Rastogi B, Kumar A, Raut SK, Panda NK, Rattan V, Joshi N and Khullar M: Downregulation of miR-377 promotes oral squamous cell carcinoma growth and migration by targeting HDAC9. Cancer Investigation 35: 152-162, 2017.

2. Bray F, Ferlay J, Soerjomataram I, Siegel RL, Torre LA and Jemal A: Global cancer statistics 2018: GLOBOCAN estimates of incidence and mortality worldwide for 36 cancers in 185 countries. CA Cancer J Clin 68: 394-424, 2018.

3. Warnakulasuriya S: Causes of oral cancer-an appraisal of controversies. Br Dent J 207: 471-475, 2009.

4. Gharat SA, Momin M and Bhavsar C: Oral squamous cell carcinoma: Current treatment strategies and nanotechnology-based approaches for prevention and therapy. Crit Rev Ther Drug Carrier Syst 33: 363-400, 2016.

5. Pollaers K, Hinton-Bayre A, Friedland PL and Farah CS: AJCC 8th edition oral cavity squamous cell carcinoma Staging-is it an improvement on the AJCC 7th edition? Oral Oncol 82: 23-28, 2018

6. Lambert R, Sauvaget C, de Camargo Cancela M and Sankaranarayanan R: Epidemiology of cancer from the oral cavity and oropharynx. Eur J Gastroenterol Hepatol 23: 633-641, 2011.

7. Yokoyama T and Nakamura T: Tribbles in disease: Signaling pathways important for cellular function and neoplastic transformation. Cancer Sci 102: 1115-1122, 2011.

8. Li K, Wang F, Cao WB, Lv XX, Hua F, Cui B, Yu JJ, Zhang XW, Shang S, Liu SS, et al: TRIB3 promotes APL progression through stabilization of the oncoprotein PML-RAR $\alpha$ and inhibition of p53-mediated senescence. Cancer Cell 31: 697-710.e7, 2017.

9. Miyoshi N, Ishii H, Mimori K, Takatsuno Y, Kim H, Hirose H, Sekimoto M, Doki Y and Mori M: Abnormal expression of TRIB3 in colorectal cancer: A novel marker for prognosis. Br J Cancer 101: 1664-1670, 2009.

10. Izrailit J, Jaiswal A, Zheng W, Moran MF and Reedijk M: Cellular stress induces TRB3/USP9x-dependent Notch activation in cancer. Oncogene 36: 1048-1057, 2017.

11. Hua F, Mu R, Liu J, Xue J, Wang Z, Lin H, Yang H, Chen X and $\mathrm{Hu} \mathrm{Z}$ : TRB3 interacts with SMAD3 promoting tumor cell migration and invasion. J Cell Sci 124: 3235-3246, 2011.

12. Wennemers M, Bussink J, Scheijen B, Nagtegaal ID, van Laarhoven HW, Raleigh JA, Varia MA, Heuvel JJ, Rouschop KM, Sweep FC and Span PN: Tribbles homolog 3 denotes a poor prognosis in breast cancer and is involved in hypoxia response. Breast Cancer Res 13: R82, 2011.

13. Zhou H, Luo Y, Chen JH, Hu J, Luo YZ, Wang W, Zeng Y and Xiao L: Knockdown of TRB3 induces apoptosis in human lung adenocarcinoma cells through regulation of Notch 1 expression. Mol Med Rep 8: 47-52, 2013.
14. Hua F, Shang S, Yang YW, Zhang HZ, Xu TL, Yu JJ, Zhou DD, Cui B, Li K, Lv XX, et al: TRIB3 interacts With $\beta$-catenin and TCF4 to increase stem cell features of colorectal cancer stem cells and tumorigenesis. Gastroenterology 156: 708-721.e15, 2019.

15. Hong B, Zhou J, Ma K, Zhang J, Xie H, Zhang K, Li L, Cai L, Zhang N, Zhang Z and Gong K: TRIB3 promotes the proliferation and invasion of renal cell carcinoma cells via activating mapk signaling pathway. Int J Biol Sci 15: 587-597, 2019.

16. Song M, Bode AM, Dong Z and Lee MH: AKT as a therapeutic target for cancer. Cancer Res 79: 1019-1031, 2019.

17. http://tcga.xenahubs.net/download/TCGA.HNSC. sampleMap/ HiSeqV2.gz.

18. Wang Y, Liu DP, Chen PP, Koeffler HP, Tong XJ and Xie D: Involvement of IFN regulatory factor (IRF)-1 and IRF-2 in the formation and progression of human esophageal cancers. Cancer Res 67: 2535-2543, 2007.

19. Vatandoost J and Kafi Sani K: A Study of recombinant Factor IX in drosophila insect $\mathrm{S} 2$ cell lines through transient gene expression technology. Avicenna J Med Biotechnol 10: 265-268, 2018.

20. Simpson DR, Mell LK and Cohen EE: Targeting the $\mathrm{PI} 3 \mathrm{~K} / \mathrm{AKT} / \mathrm{mTOR}$ pathway in squamous cell carcinoma of the head and neck. Oral Oncol 51: 291-298, 2015.

21. Zhang J, Wen HJ, Guo ZM, Zeng MS, Li MZ, Jiang YE, He XG and Sun CZ: TRB3 overexpression due to endoplasmic reticulum stress inhibits AKT kinase activation of tongue squamous cell carcinoma. Oral Oncol 47: 934-939, 2011.

22. Wennemers M, Bussink J, Grebenchtchikov N, Sweep FC and Span PN: TRIB3 protein denotes a good prognosis in breast cancer patients and is associated with hypoxia sensitivity. Radiother Oncol 101: 198-202, 2011.

23. Revathidevi S and Munirajan AK: Akt in cancer: Mediator and more. Semin Cancer Biol 59: 80-91, 2019.

24. Ikink GJ, Boer M, Bakker ER and Hilkens J: IRS4 induces mammary tumorigenesis and confers resistance to HER2-targeted therapy through constitutive PI3K/AKT-pathway hyperactivation. Nat Commun 7: 13567, 2016.

25. Ediriweera MK, Tennekoon KH and Samarakoon SR: Role of the PI3K/AKT/mTOR signaling pathway in ovarian cancer: Biological and Therapeutic Significance. Semin Cancer Biol 59: 147-160, 2019.

26. Restelli M, Magni M, Ruscica V, Pinciroli P, De Cecco L, Buscemi G, Delia D and Zannini L: A novel crosstalk between CCAR2 and AKT pathway in the regulation of cancer cell proliferation. Cell Death Dis 7: e2453, 2016.

This work is licensed under a Creative Commons Attribution-NonCommercial-NoDerivatives 4.0 International (CC BY-NC-ND 4.0) License. 